\title{
Alpha EEG correlates of performance on a music recognition task
}

\author{
JAMES L. WALKER \\ Brandon University, Brandon, Manitoba R7A 6A9, Canada
}

\begin{abstract}
EEGs were recorded over left and right occipital sites in 12 right-handed, musically naive subjects during the presentation of a series of piano melodies. The subjects were required to detect melodies presented earlier in the series. Sample epochs were subjected to a period analysis for alpha, theta, and delta waves, and EEG frequency was related to recording site, ear stimulated, and performance on the recognition task. The incidence of alpha waves by period analysis was found to significantly differentiate between positive and negative responses to the stimuli. No significant hemispheric lateralization or ear effects were obtained.
\end{abstract}

Over the past several years, there has been an increasing interest in electroencephalographic indices of cerebral lateralization in the human brain. A typical research paradigm has involved recording the EEG over left and right hemispheric sites during the presentation of tasks thought to involve primarily the left or the right hemisphere. In these studies, the presentation of a musical task has frequently been used in an attempt to provide a task engaging primarily the right hemisphere (Davidson \& Schwartz, 1977; Doyle, Ornstein, \& Galin, 1974; McKee, Humphrey, \& McAdam, 1973; Osborne \& Gale, 1976; Ray, Morrell, Frediani, \& Tucker, 1976). The use of a musical task as a "right-hemisphere" task rests upon work by Kimura (1964) indicating that, under conditions of dichotic stimulation, subjects show superior performance scores to stimuli presented to the left ear. Accepting the common view that contralateral auditory projections are dominant over ipsilateral pathways, this finding supports the position that the right hemisphere is superior to the left in processing melodic stimuli. Bever and Chiarello (1974) have reported that lateralization effects in processing musical stimuli can also be demonstrated under conditions of monaural stimulation and have suggested that the traditional left-ear superiority is observed only in musically naive subjects, with musically sophisticated subjects showing a right-ear superiority in music recognition tasks and hence a presumed lefthemisphere superiority.

While a number of studies in the EEG literature have reported interhemispheric alpha differences consistent with the hypothesis that music processing involves the right more than the left hemisphere,

This study was supported by Brandon University President's Research Grant 2374. Thanks are extended to Katherine L. Colquhoun for assistance in conducting the study. Requests for reprints may be addressed to the author, at Department of Psychology, Brandon University, Brandon, Manitoba R7A 6A9, Canada. no studies exist that specifically investigate the relationship between EEG measures of brain activity and performance on a music processing task. The existing literature reflects a situation in which traditional dichotic and monaural stimulation studies have monitored performance but not brain activity, while typical EEG research has recorded brain activity without relating it to the behavioral performance on the music task. The purpose of the present study was to investigate interhemispheric differences in alpha, theta, and delta activity during a music-recognition task and to study the relationship between these electrophysiological measures and how subjects performed in a music-recognition task.

\section{METHOD}

\section{Subjects}

Twelve undergraduate students attending summer classes at Brandon University participated in the study. All subjects were unpaid volunteers who were told that the research would involve a "study of brain activity during a music recognition task." Prior to the experiment, each student was given a hand-preference questionnaire (Lomas \& Kimura, 1976) and any student showing a left-hand preference for any activity listed on the questionnaire was not included in the study. Equal numbers of males and females were represented; their ages ranged from 17 to 43 years, with a mean age of 28 years. None of the subjects reported having taken formal instruction in music or having played a musical instrument within the previous 5 years.

\section{Procedures}

EEG recordings were collected during a melody-recognition task similar to that used by Bever and Chiarello (1974). The task consisted of the monaural presentation of a series of 5-sec excerpts from Beethoven piano sonatas (Deutsche Grammaphon, STL-44, $2563,033-037)$. At the end of each excerpt, the subjects were instructed to report verbally whether the excerpt had been presented earlier in the session. All instruments and melodic stimuli were recorded in the monaural mode, and the tape was played at a constant comfortable listening level over a stethoscope headset. Half of the subjects heard the auditory stimuli only at the left ear, while the remaining subjects heard the stimuli at the right ear. The first seven trials were practice trials during which the subjects were presented with the correct answer prior to the begin- 
ning of the next practice trial. The remaining 48 trials were experimental trials, and the subjects were not informed of the correctness or incorrectness of any of their responses to these trials. Each music segment was $5 \mathrm{sec}$ long, and successive stimuli were separated by a 10 -sec silent period during which subjects responded. The total duration of the recognition task, exclusive of the practice trials, was $12 \mathrm{~min}$. Of the 48 experimental trials, 12 represented excerpts that had been presented previously. From one to six trials intervened between the first and second presentation of a repeated melody. No segment was repeated more than once. All stimuli were presented using the same channel of the tape deck and through the same speaker of the headset, which was positioned on the subject so that the appropriate ear received the sound.

Grass gold cup electrodes were attached with collodion over left- and right-hemisphere occipital locations at $\mathrm{O}_{1}$ and $\mathrm{O}_{2}$ of the 10-20 International Electrode Placement system. Prior to recording, the impedance of each electrode was tested, and any showing an interelectrode impedance of $8 \mathrm{k} \Omega$ or larger was reapplied until the impedance was smaller than this value. Recordings were bipolar, with each electrode referenced to a vertex electrode at $\mathrm{C}_{2}$. The ground electrode was placed on the right ear lobe. Additional electrodes were positioned over the outer canthi of each eye and above and below the left eye to record lateral and vertical eye movements (EOG). All recordings were done on a Grass Model 79 polygraph, with the EEG amplifiers calibrated so that a $50-\mu \mathrm{V}$ signal produced a $1-\mathrm{cm}$ pen deflection. One-half-amplitude low attenuation of each amplifier was set at $.1 \mathrm{~Hz}$, and the experiment was conducted with the line filters in. Amplifiers were recalibrated before each recording session. The chart speed was set at $30 \mathrm{~mm} / \mathrm{sec}$ and the onset and offset of each auditory stimulus was marked on the chart using the polygraph signal marker.

During the experiment, the subjects sat, with their eyes closed, in an upright position in a recliner chair in a sound-deadened room. An experimenter was also in the room throughout each session to record the subjects' responses to each music stimulus.

Prior to conducting the analysis, any EEG epochs that coincided with EOG activity were edited and were not included in the final EEG frequency analysis. EEG records were scored using the manual period analysis method developed by Sulg (1969). Successive wave troughs were used as reference points for the demarcation of separate EEG waves and all clearly visible waves including superimposed waves of amplitudes of at least $5 \mu \mathrm{V}$ were designated by drawing vertical lines through the wave troughs. A transparency multiscale (Sulg, 1969) was then used to determine the frequency of each EEG wave within a frequency band of 1-13 Hz. Because of practical restriction, it was not possible to analyze all of the EEG records collected during the course of the study. A table of random numbers was used to select six repeated trials from the 12 present in the stimulus series and to select another six nonrepeated trials from the 36 represented in the series. These 12 epochs were included in the final EEG frequency analysis. The repated melodies occurred on Trials $4,8,12,28,35$, and 48, while the nonrepeated melodies occurred on Trials $5,9,27$, 34,37 , and 41 . For each of these epochs, measures were collected describing the percent activity time of three EEG frequency classes. Percent alpha represented percent time of $8-13 \mathrm{~Hz}$ activity, percent theta reflected 4-7 Hz activity, and percent delta was defined as 1-3 Hz EEG frequencies. Three frequency measures were thus represented for each analyzed EEG epoch: percent activity time alpha, theta, and delta.

At the conclusion of the experiment, each subject's response record on the music-recognition task was scored. The experimenter who completed the scoring was not the experimenter who conducted the EEG analysis. The recognition-task scoring was thus independent and blind with respect to the EEG frequency analysis. Every response on the music-recognition task was classified as representing a true positive (TP), true negative (TN), false positive
(FP), or false negative (FN) response. A total score on the test, corrected for guessing, was calculated by subtracting one-half the number of incorrect responses expressed as a proportion of total possible incorrect responses from the number of correct responses expressed as a proportion of total possible correct responses [total score $=(\mathrm{TP} / 12+\mathrm{TN} / 36)-1 / 2(\mathrm{FN} / 36+\mathrm{FN} / 12)]$. This corrected performance score on the total 48-trial task was used solely to determine if differences were present between subjects who had the music presented at different ears. The performance classification used for all EEG analyses was based entirely upon the randomly selected 12 trials which received the EEG frequency analysis. For purposes of the EEG analyses, the percent activity times in each bandwidth was summed for each of the 12 EEG epochs which represented a TP response. This summation of percent activity times was then divided by the number of TBs occurring for that subject within the 12 sampled trials, thus representing a mean percent EEG activity time for TP responses. A similar procedure was used to determine a mean percent EEG activity time for all FP responses and for all TN responses. A mean percent EEG activity time for FN responses was not calculated, since the number of FN responses across subjects was too small to obtain meaningful estimates of this value. The following dependent variables were thus obtained and used in subsequent analyses: mean percent activity times alpha for TPs, FPs, and TNs; mean percent activity times theta for TPs, FPs, and TNs; and mean percent activity times delta for TPs, FPs, and TNs.

At the completion of the frequency analysis, all EEG measures were punched onto computer cards and analyses of variance were computed using computer program BMDP1V (Dixon, 1975). The ANOVA design represented a 2 by 2 by 3 factorial (ear stimulated by recording site by performance category). Two-tailed t tests were used to test the significance of individual comparisons.

\section{RESULTS}

To determine if subjects performed better on the music recognition task under conditions of left-ear stimulation as compared with right-ear stimulation, a $t$ test was conducted on the total recognition-task scores, corrected for guessing, between subjects hearing the task in the left ear compared with subjects hearing the music in the right ear. A perfect score with no errors would yield a maximum score of 2.0. The mean total performance score for subjects hearing the music at the left ear was 1.26 , and the mean score for subjects hearing the music at the right was 1.22. These means did not significantly differ from each other $[\mathrm{t}(11)=.25, \mathrm{p}<.82]$.

The analysis of variance on the mean alpha activity time scores produced a significant main effect for the performance variable $[F(2,40)=3.44, p<.04]$. The mean percent alpha for TP trials was 46.7 , for FP trials, 47.6 and for TN trials 54.6. Two-tailed t tests revealed a significant difference between TP and TN responses $[\mathrm{t}(23)=2.36, \mathrm{p}<.03]$, indicating more alpha activity during the TN than during the TP categories. A significant difference between FP and TN categories was also obtained using two-tailed t tests $[\mathrm{t}(23)=2.56$, $\mathrm{p}<.02$ ], indicating more alpha activity during TN trials than during FP trials. It is noteworthy that a $t$ test between TP and FP alpha times revealed no significant differences between the two categories. F 
Table 1

Means and Standard Deviations of Alpha Scores

\begin{tabular}{cccccccccc}
\hline & \multicolumn{9}{c}{ Site } \\
\cline { 2 - 9 } & \multicolumn{4}{c}{ Left Ear } & \multicolumn{3}{c}{ Right Ear } \\
\cline { 2 - 9 } Performance & \multicolumn{2}{c}{$\mathrm{O}_{1}$} & \multicolumn{2}{c}{$\mathrm{O}_{2}$} & \multicolumn{2}{c}{$\mathrm{O}_{1}$} & \multicolumn{2}{c}{$\mathrm{O}_{2}$} \\
\cline { 2 - 9 } Category & Mean & SD & Mean & SD & Mean & SD & Mean & SD \\
\hline True Positive & 44.5 & 12.2 & 46.2 & 17.1 & 42.9 & 20.8 & 53.3 & 14.1 \\
False Positive & 50.7 & 23.2 & 49.4 & 18.1 & 45.2 & 21.9 & 45.3 & 13.3 \\
True Negative & 52.2 & 16.9 & 50.9 & 17.6 & 59.3 & 13.8 & 56.2 & 13.5 \\
\hline
\end{tabular}

Table 2

Means and Standard Deviations of Theta Scores

\begin{tabular}{lrrrrrrrr}
\hline & \multicolumn{9}{c}{ Site } \\
\cline { 2 - 9 } & \multicolumn{4}{c}{ Left Ear } & \multicolumn{4}{c}{ Right Ear } \\
\cline { 2 - 9 } Performance & \multicolumn{2}{c}{$\mathrm{O}_{1}$} & \multicolumn{2}{c}{$\mathrm{O}_{2}$} & \multicolumn{2}{c}{$\mathrm{O}_{1}$} & \multicolumn{2}{c}{$\mathrm{O}_{2}$} \\
\cline { 2 - 9 } Category & Mean & SD & Mean & SD & Mean & SD & Mean & SD \\
\hline True Positive & 10.9 & 2.8 & 9.5 & 5.1 & 8.8 & 1.8 & 9.3 & 5.3 \\
False Positive & 8.7 & 6.7 & 8.6 & 6.6 & 18.5 & 28.3 & 10.6 & 6.9 \\
True Negative & 12.3 & 8.2 & 10.0 & 4.2 & 20.4 & 28.2 & 11.0 & 4.5 \\
\hline
\end{tabular}

ratios for the main effects of ear and of site were both less than one, and none of the interaction terms were significant [Ear by Site, $F(1,20)=.06$; Ear by Performance, $F(2,40)=1.47, p<.25$; Site by Performance, $F(2,40)=.89$; Ear by Site by Performance, $F(2,40)=.33$. Means and standard deviations of scores are presented in Table 1.

No significant $F$ ratios were obtained in analyses conducted on mean theta or mean delta activity time scores, indicating that these dependent measures did not differ as a function of how the subjects performed on the recognition task. Means and standard deviations on theta scores are presented in Table 2.

\section{DISCUSSION}

The results of this study suggest that, during a melodic recognition task, alpha activity at occipital sites is related to the subsequent decision reached by the subject in an information processing task. Significant differences were obtained in the mean alpha EEG activity observed during the presentation of melodic stimuli that were correctly classified by the subjects as being repetitions (TPs) as compared with those stimuli that were correctly classified as not representing repetitions (TNs). The occurrence of more alpha activity during the correctly classified negative trials than during the correctly classified positive trials suggests that subject classification of a stimulus as a positive trial may be associated with higher levels of cortical activation than are observed during trials subsequently classified by subjects as negative. Interestingly, the amount of cortical activa- tion as reflected by alpha activity was similar whenever the subjects subsequently classified a stimulus as a repetition, independent of whether this response was objectively correct (TP) or incorrect (FP).

Studies using perceptual processing or auditory vigilance tasks bearing some similarity to the task in the present study present contradictory findings regarding the relationship between EEG activity and correct or incorrect responses. Daniel (1967), for example, found that, in an auditory vigilance task in which subjects were asked to detect certain critical sequences of digits, the incidence of alpha activity did not distinguish between detections and errors but that the incidence of theta activity decreased prior to failures in detecting a critical stimulus. Results discrepant with those of Daniel (1967) have been reported by Haslum and Gale (1973). Using both auditory and visual stimuli and requiring subjects to respond when a critical sequence of digits was presented, it was found that as an auditory signal more closely approximated the critical stimulus sequence, alpha activity decreased while the incidence of theta activity increased. Similar results were obtained by Gale, Haslum, and Lucas (1972), using only auditory stimuli. The finding of the present study that alpha activity decreased significantly during stimuli classified as positive instances as compared with the occurrence of higher levels of alpha activity during stimuli classified as negative trials is consistent with the Gale et al. (1972) and Haslum and Gale (1973) findings, although no significant changes in theta activity were observed in the present study.

The present data on alpha differences provides additional support for activation theory (Lindsley, 1960). From activation theory, it might be argued that, during the presentation of a musical stimulus that the subject initially classifies as a possible repetition, arousal level would be heightened as each successive segment of the stimulus was judged to match a previously presented stimulus. Activation and expectancy might then be progressively heightened as each successive melody segment was judged to match a previous melody. In the case of melodies judged not to represent repetitions, it is possible that the initial judged mismatch between the melody and memories of previous melodies is associated with greater relaxation, which continues as more of the melody is presented and is judged not to represent a match to a previous melody. In effect, after the initial melody segment is judged to be a mismatch, the subject can relax since, at that point, the demand characteristics of the recognition process have essentially ended. This interpretation is consistent with the findings of Gale et al. (1972) that, as a sequence of digits progressively approximated that of the critical stimulus sequence, increased signs of EEG activation were observed.

In previous work from our laboratory (Walker, 
1977), self-reports of attentiveness while listening to music were found to correlate significantly with both occipital theta and alpha. These correlations suggested that increased theta production was associated with decreased attention, while increases in alpha were associated with increases in self-reports of attentiveness. These results are not generally consistent with the finding and interpretations of the present experiment. Although theta was found to be inversely correlated with self-reports of attentiveness in the earlier study, the present experiment failed to find theta differences between trials classified by subjects as repetitions vs. nonrepetitions. If, as hypothesized in the present work, trials representing repetitions were associated with increased attentiveness, the earlier study would predict a decrease in theta during such trials (TP and FP trials). These differences were not observed in the present experiment. The alpha correlation reported in Walker (1977), in which increased alpha was found to show a moderate correlation with self-reports of increased attentiveness to the music, also poses some conceptual difficulties in the interpretation of the present results, since the direction of the correlation differs from the present findings of less alpha during trials classified as positive-those trials during which we have hypothesized increases in attentiveness. Although both Walker (1977) and the present work suggest a possible relationship between alpha activity and attentiveness during music listening, the nature of the relationship remains unclear. It is possible that some of the discrepancy may be due to differences in the tasks used in the two studies. Walker (1977) had subjects passively listen to two musical selections and subsequently report on their subjective reactions to the music. The task used in the present experiment involved demand characteristics which required making sophisticated discriminations among a large selection of musical stimuli. Such a task appears more similar to that used by Haslum and Gale (1973), in which alpha results consistent with the present results were reported, than to the passive listening used in the Walker (1977) study. It would be instructive in future work to use a task with demand characteristics and also to allow for immediate measures of attentiveness, including both self-reports and other physiological measures.

Bever and Chiarello (1974) reported that lateralization effects in processing musical stimuli can be demonstrated under conditions of monaural stimulation similar to those used in the present study. They report that musically naive subjects show a left-ear superiority, whereas musically sophisticated subjects show a right-ear superiority. Using a paradigm similar to that of Bever and Chiarello, the present study failed to demonstrate superior left-ear performance in a group of musically naive subjects. The present study also failed to detect EEG frequency laterality effects during a music-recognition task. No significant left-right differences in percent alpha, theta, or delta were observed, nor did the site of recording interact with ear stimulated or performance score, as might have been expected had the two hemispheres been differentially involved in processing the melodic stimuli.

\section{REFERENCES}

Bever, T. G., \& Chiarello, R. J. Cerebral dominance in musicians and nonmusicians. Science, 1974, 185, 537-539.

Daniel, R. S. Alpha and theta EEG in vigilance. Perceptual and Motor Skills, 1967, 24, 697-703.

Davidson, R. J., \& Schwartz, G. E. The influence of musical training on patterns of EEG asymmetry during musical and non-musical self-generation tasks. Psychophysiology, 1977, 14, 58-63.

Doyle, J. C., Ornstein, R., \& Galin, D. Lateral specialization of cognitive mode: II. EEG frequency analysis. Psychophysiology, 1974, 11, 567-578.

Dixon, W. J. (Ed.). Biomedical computer programs. Berkeley: University of California Press, 1975.

Gale, A., Haslum, M., \& Lucas, B. Arousal value of the stimulus and EEG abundance in an auditory vigilance task. British Journal of Psychology, 1972, 63, 515-522.

HASLUM, M. H., \& GALE, A. Inter-modal and intra-subject consistency in EEG correlates of vigilance. Biological Psychology, 1973, 1, 139-150.

Kimura, D. Left-right differences in the perception of melodies. Quarterly Journal of Experimental Psychology, 1964, 16, 355-358.

LindSLEY, D. B. Attention, consciousness, sleep and wakefulness. In J. Field, H. W. Magoun, \& V. E. Hall (Eds.), Handbook of physiology (Vol. 3). Washington, D.C: American Physiological Society, 1960.

Lomas, J., \& Kimura, D. Intrahemispheric interaction between speaking and sequential manual activity. Neuropsychologia, 1976, 14, 23-33.

McKee, G., Humphrey, B., \& McAdam, D. W. Scaled lateralization of alpha activity during linguistic and musical tasks. Psychophysiology, 1973, 10, 441-443.

Osborne, K., \& GALe, A. Bilateral EEG differentiation of stimuli. Biological Psychology, 1976, 4, 185-196.

Ray, W. J., Morrell, M., Frediani, A. W., \& Tucker, D. Sex differences and lateral specialization of hemispheric functioning. Neuropsychologia, 1976, 14, 291-394.

Sulg, I. A. Manual EEG analysis. Acta Neurologica Scandinavica, 1969, 45, 431-458.

Walker, J. L. Subjective reactions to music and brainwave rhythms. Physiological Psychology, 1977, 5, 483-489.

(Received for publication March 7, 1978; revision accepted April 18, 1980.) 